\title{
Stress Dose Steroid Therapy
}

National Cancer Institute

\section{Source}

National Cancer Institute. Stress Dose Steroid Therapy. NCI Thesaurus. Code C121368.

The administration of corticosteroids to an individual with either a long term history of corticosteroid use, or a history of or increased risk for adrenal insufficiency during times of increased physiological stress, including surgery or serious illness. 